\title{
Pemphigoid gestationis: Report of 44 cases
}

\section{Amina Aounallah ${ }^{1,2}$, Ines Lahouel ${ }^{1,2}$, Lobna Boussofara ${ }^{1,2}$, Sana Mokni ${ }^{1,2}$, Najet Ghariani ${ }^{1,2}$, Mouna Korbi ${ }^{1,2}$, Wafa Saidi ${ }^{1,2}$, Badreddine Sriha ${ }^{1,3}$, Mohamed Denguezli ${ }^{1,2}$, Colandane Belajouza ${ }^{1,2}$, Rafiaa Nouira ${ }^{1,2}$}

${ }^{1}$ Sousse University, Sousse, Tunisia, ${ }^{2}$ Dermatology Department of Farhat Hached Hospital, Sousse, Tunisia, ${ }^{3}$ Anatomopathology Department of Farhat Hached Hospital, Sousse, Tunisia

Corresponding author: Dr. Ines Lahouel, E-mail: Lahouel.iness@gmail.com

\begin{abstract}
Introduction: Pemphigoid gestationis (PG) is a rare autoimmune bullous dermatosis occurring during pregnancy and post partum. The aim of our study was to analyse epidemio-clinical, immunopathological, therapeutic and prognostic features of PG through a hospital study of 44 cases. Methods: It is a retrospective study, collecting all cases diagnosed at the dermatological department of Farhat Hached Soussa (Tunisia) from January 1990 to December 2015. Results: The disease began in 40,9\% of cases at the second trimester of pregnancy and 48\% at the third trimester of pregnancy, one case in post abortum. It was a polymorphic eruption, localized in the chest, the back and limbs in all cases. The face was affected in 18,2\% of cases and the mucosa in one case. The skin biopsy showed a subepidermal blister in 25 cases. The direct immunofluoresence found linear C3 deposits along the basement membrane in all cases, associated to IgG deposits in $25 \%$ of cases. The treatment was mainly based on local or general corticotherapy with favourable evolution. We noted exacerbation in post partum in 17 cases and relapses during the ulterior pregnancies in 7 cases. Fetal damage was present in PG in 8 cases. Conclusion: Our study is conformed to literature data; nevertheless, it is distinguished by its appearance on the face the less frequency of palmo-plantar manifestations and the exceptional case of PG postabortum.
\end{abstract}

Key words: Pemphigoid gestationis; Bullous disease; Pregnancy; Descriptive study

How to cite this article: Aounallah A, Lahouel I, Boussofara L, Mokni S, Ghariani N, Korbi M, Saidi W, Sriha B, Denguezli M, Belajouza C, Nouira R. Pemphigoid gestationis: Report of 44 cases. Our Dermatol Online. 2017;8(3):255-259.

Submission: 21.11.2016; Acceptance: 27.02.2017

DOI: 10.7241/ourd.20173.74 


\title{
Pemphigoïde gestationis: Revue de 44 cas
}

\section{Amina Aounallah ${ }^{1,2}$, Ines Lahouel ${ }^{1,2}$, Lobna Boussofara ${ }^{1,2}$, Sana Mokni ${ }^{1,2}$, Najet Ghariani ${ }^{1,2}$, Mouna Korbi ${ }^{1,2}$, Wafa Saidi ${ }^{1,2}$, Badreddine Sriha ${ }^{1,3}$, Mohamed Denguezli ${ }^{1,2}$, Colandane Belajouza ${ }^{1,2}$, Rafiaa Nouira ${ }^{1,2}$}

\author{
${ }^{1}$ Sousse University, Sousse, Tunisia, ${ }^{2}$ Dermatology Department of Farhat Hached Hospital, Sousse, Tunisia, \\ ${ }^{3}$ Anatomopathology Department of Farhat Hached Hospital, Sousse, Tunisia
}

Corresponding author: Dr. Ines Lahouel, E-mail: Lahouel.iness@gmail.com

\begin{abstract}
RESUME
Introduction: La pemphigoïde gestationis est une dermatose bulleuse auto immune rare de la grossesse et du postpartum. Notre but est d'analyser les aspects épidémio-cliniques, immunopathologiques, thérapeutiques et pronostiques de la PG à travers une série hospitalière de 44 cas. Materiels et Methodes: Il s'agit d'une étude rétrospective, colligeant les cas de PG diagnostiqués au service de dermatologie de l'hôpital Farhat Hached de Sousse (Tunisie) entre Janvier 1990 et Décembre 2015. Resultats: Nous avons colligéen 35 multipares et 9 primipares. La maladie débutait dans $40,9 \%$ des cas au $2^{\text {ième }}$ trimestre de gestation et $48 \%$ des cas au $3^{\text {ième }}$ trimestre, avec un cas de PG post abortum. Elle était polymorphe, localisée au niveau du tronc et des membres dans tous les cas. Le visage était atteint dans 18,2\% des cas et une atteinte muqueuse chez une patiente. Lexamen histologique montrait une bulle sous épidermique dans 30 cas. Limmunofluorescence directe retrouvait un dépôt de C3 le long de la membrane basale dans tous les cas associé à un dépôt d'IgG dans $25 \%$ des cas. Le traitement était basé sur la corticothérapie avec une évolution favorable. Des exacerbations en post partum ont été notées chez 17 patientes et des récidives lors des grossesses ultérieures chez 7 autres. Un retentissement foetal a été recensé dans 8 cas. Conclusion: Notre série se caractérise par la fréquence de l'atteinte du visage, la rareté de l'atteinte palmoplantaire et l'exceptionnel cas de PG postabortum.
\end{abstract}

Palabras claves: Mots clés:Pemphigoïde gestationis; Maladie bulleuse auto immune; Grossesse; Étude descriptive

\section{INTRODUCTION}

Décrite depuis 1872, sous la dénomination d'herpès gestationis, la pemphigoïde gestationis (PG) est une dermatose bulleuse auto-immune (DBAI) de la grossesse et du post-partum $[1,2]$.

Le but de notre travail est d'analyser les différents aspects épidémio-cliniques, immuno-pathologiques, thérapeutiques et pronostiques de la PG à travers une série hospitalière de 44 cas.

\section{MATERIELS ET METHODES}

Il s'agit d'une étude rétrospective, colligeant tous les cas de PG hospitalisés au service de dermatologie de l'hôpital Farhat Hached de Sousse (Tunisie) sur une période de 26 ans (1990-2015).
Pour chaque patiente, les données suivantes étaient recueillies: âge, antécédents d'épisodes antérieurs, nombre de grossesses, parité, terme de la grossesse, manifestations cliniques et résultats des examens complémentaires biologiques, de la biopsie cutanée, et de l' l'immunofluorescence directe (IFD) et indirecte (IFI), les modalités thérapeutiques et évolutives, l'existence d'un retentissement materno-foetal et la survenue d'une récidive ultérieure.

\section{RESULTATS}

Quarante quatre cas de PG ont été recensés. Lâge moyen était de 32,43 ans (20 ans - 41 ans). La majorité (79,5\% des cas) étaient multipares. La PG débutait dans $56 \%$ des cas au cours des trois premières grossesses, au deuxième trimestre pour 18 patientes $(40,9 \%)$, au troisième trimestre pour $21(48 \%)$ et au premier

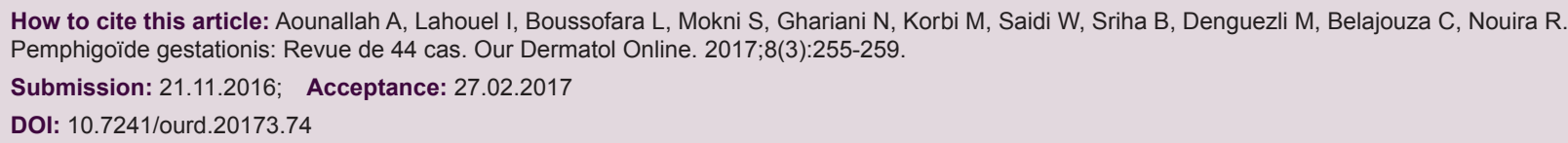


trimestre pour 2 (4,5\%). Deux patientes ont présenté des lésions bulleuses en post-partum $(4,5 \%)$ et une en post-abortum $(2,1 \%)$.

Le prurit était constant. Le site initial des lésions était la région périombilicale pour 31 patientes $(70,4 \%)$ et les membres pour les 13 restantes (29,6\%). Léruption était le plus souvent polymorphe. Des lésions pseudourticariennes étaient observées chez 39 patientes $(88,6 \%)$ (Fig. 1), des vésicules chez 34 (77,2\%), des bulles chez $25(68,1 \%)$, des cocardes chez $7(15,9 \%)$ (Fig. 2) et des pustules chez 8 (18,2\%). Léruption était étendue au tronc et aux membres dans tous les cas. L'atteinte du visage était notée chez huit patientes (18,2\% des cas). Trois atteintes palmoplantaires $(6,8 \%)$ ont été retrouvées (Fig. 3). Lexamen des muqueuses a objectivé des érosions buccales chez une seule patiente.

Les examens biologiques révélaient une hyperleucocytose $\left(\geq 10000\right.$ éléments $/ \mathrm{mm}^{3}$ ) dans 22 cas $(50 \%)$ et une hyperéosinophilie ( $\geq 500$ éléments $/ \mathrm{mm}^{3}$ ) dans 25 cas (56,8\%). La vitesse de sédimentation était accélérée dans 12 cas $(\mathrm{Hl} \geq 50)(27,2 \%)$.

L'histologie montrait dans tous les cas un œè̀me du derme papillaire associé à un infiltrat lymphohistiocytaire riche en polynucléaires éosinophiles. Des bulles sous épidermiques ont été mises en évidence dans 30 cas (68,18\%); l'IFD était positive dans tous les cas. L'IFI, pratiquée uniquement chez deux patientes, était négative.

Le traitement reposait sur la corticothérapie générale, utilisée efficacement chez 32 patientes $(72,72 \%)$ à des doses allant de 0,5 à $1 \mathrm{mg} / \mathrm{kg} / \mathrm{j}$. Les dermocorticoïdes forts associés à un traitement antihistaminique ont permis la cicatrisation des lésions bulleuses chez 10 patientes. Une patiente a bien répondu à la disulone débutée au deuxième jour du post partum, à la dose de $200 \mathrm{mg} / \mathrm{j}$ avec guérison des lésions au bout de 5 semaines. Seule une patiente présentant une éruption moyennement étendue n’a pas été améliorée par l'utilisation des dermocorticoïdes et a nécessité sa mise sous corticothérapie générale avec une bonne évolution.

Le délai de guérison était en moyenne de 5 semaines (2 semaines - 20 semaines). Un retentissement foetal a été recensé dans 8 cas $(18,18 \%)$ à type de: mort foetale in utéro dans un cas, prématurité dans 2 cas $(4,5 \%)$, hypotrophie dans 3 cas $(6,8 \%)$ et de lésions néonatales vésiculo-bulleuses transitoires survenant à $\mathrm{J} 2$ et à $\mathrm{J} 5$ de vie dans deux autres cas $(4,5 \%)$.

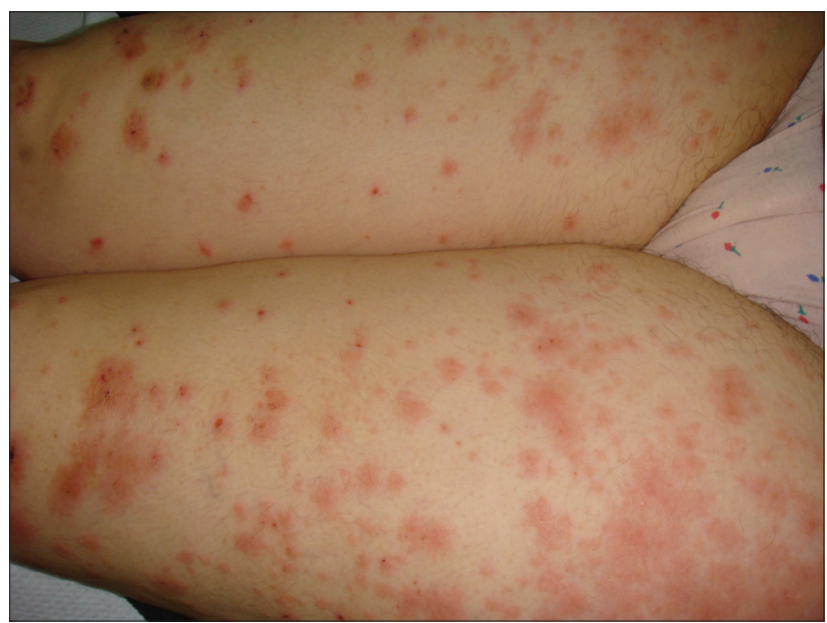

Figure 1: Plaques érythémateuses urticariennes des cuisses.

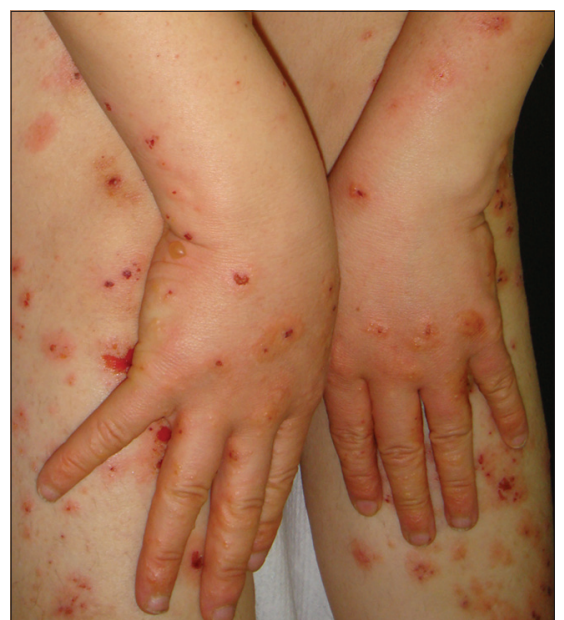

Figure 2: Eruption vésiculo-bulleuse généralisée avec par endroit un aspect en cocarde.

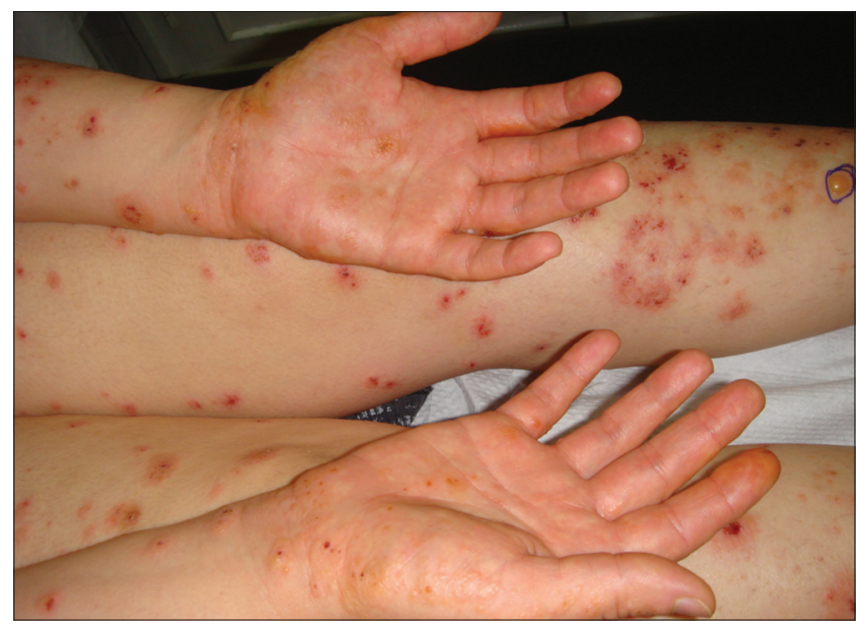

Figure 3: Atteinte palmaire au cours d'une pemphigoïde gestationis.

En post partum, des exacerbations des lésions cutanées ont été observées chez 17 patientes $(38,6 \%)$ dont 10 étaient sous dermocorticoïdes et 7 sous corticothérapie 
générale. Sept des 29 patientes qui ont été revues au cours des grossesses ultérieures ont développé une récidive.

\section{DISCUSSION}

La pemphigoïde gestationis (PG) est une DBAI sous épidermique rare induite par la grossesse. Son incidence dans le monde varie entre $1 / 10000$ [3] à $1 / 50000$ [4] grossesses. Elle semble plus fréquente en Tunisie (1/6000 grossesses) [5].

La cible des autoanticorps est l'Ag BPAg2 qui est une protéine transmembranaire de 180 kDa. L'Ag BPAg2 existe au niveau de la jonction dermo-épidermique et la jonction chorio-amniotique à partir du deuxième trimestre. Au cours de la PG, il se produit une rupture de tolérance vis-à-vis de cet antigène chez des femmes prédisposées génétiquement (groupes HLA DR3, DR4 et C4Q0) [5-8].

La PG touche la femme jeune entre 20 et 40 ans [6]. Elle survient le plus souvent chez les multipares [4], comme les résultats de notre série.

La PG peut apparaître pour la première fois pendant n'importe quelle grossesse, mais le plus souvent le début survient au cours des trois premières grossesses (63 à 75\% des cas), comme les résultats de notre série. La PG apparaît souvent au cours du deuxième ou troisième trimestre de la gestation $[4,8]$. Dans notre série, le pic de survenue était au deuxième trimestre.

Cependant, nous avions noté un cas exceptionnel de PG survenu la première fois à $\mathrm{J} 2$ du post-abortum. Il s'agissait d'une forme sévère ayant récidivé au cours des trois grossesses ultérieures et s'est compliquée de mort foetale in utero. A notre connaissance il n'y a aucune publication qui a rapporté ce genre de fait.

Cliniquement la PG débute par un prurit insomniant [4] suivi par l'apparition de lésions maculo-papuleuses confluentes en placards parfois circinés [8]. En 2 à 4 semaines, ces plaques vont se couvrir de vésicules et/ou bulles pouvant donner un aspect en cible ou en cocarde. Plus rarement, des lésions pustuleuses peuvent être observées [4]. Dans notre série, la fréquence des lésions pustuleuses était relativement élevée,

Le site initial de l'éruption est le plus souvent la région ombilicale/péri ombilicale[8]. Le visage et les muqueuses sont classiquement respectés [9]. Notre série se distingue par une fréquence élevée d'atteinte du visage $(18,2 \%)$. Par ailleurs, nous avons également noté la faible fréquence de l'atteinte palmoplantaire alors qu'elle est fréquemment rapportée dans la littérature [4,5].

Classiquement, l'histologie montre un décollement sous épidermique sans nécrose du toit ni acantholyse associé à un infiltrat inflammatoire lymphohistiocytaire du derme papillaire riche en éosinophiles [4]. Nous avons retrouvé un aspect typique de bulle sous épidermique dans 68,18\% [10].

Le diagnostic de $\mathrm{PG}$ est confirmé par la présence à l'IFD d'un dépôt linéaire constant de $\mathrm{C} 3$ au niveau de la membrane basale associé ou non à un dépôt d'IgG dans 25 à $40 \%$ des cas [4,8]. Dans notre série, l'IFD était positive chez toutes nos patientes, montrant un dépôt de C3 le long de la membrane basale dans $100 \%$ des cas associé à un dépôt d'IgG dans $25 \%$ des cas. L'IFI montre la présence dans le sérum des patientes, d'IgGl fixant le complément et dirigés contre la membrane basale, détectés dans 60 à $90 \%$ des cas [11]. Cet examen n'a pas été pratiqué que chez 2 de nos patientes et revenu négatif. Limmunotransfert reconnaît deux antigènes principaux le premier de $180 \mathrm{Kd}$ reconnu par $70 \%$ des autoanticorps et le deuxième de $230 \mathrm{Kd}$ reconnu par $30 \%$ des autoanticorps de la PG $[5,12]$. Limmunomicroscopie électronique détecte les dépôts de $\mathrm{C} 3$ et IgG dans la lamina lucida, le long du pôle basal des kératinocytes [5]. Cet examen n'est pas de pratique courante, d'ailleurs dans notre étude cette technique n'a pas été faite.

Sur le plan thérapeutique, les formes peu étendues de la $\mathrm{PG}$ répondent favorablement à une corticothérapie locale associée à des antihistaminiques [5,12]. Quant aux formes sévères, une corticothérapie générale est indiquée à la dose de 0,5 à $1 \mathrm{mg} / \mathrm{kg} / \mathrm{J}$ [8]. La disulone pourrait être une alternative intéressante [4].

Dans notre série, la corticothérapie générale a été largement utilisée sans incidence sur le suivi des grossesses. Les poussées du post-partum étaient notées chez 38,6\% de nos patientes ce qui est relativement peu fréquent par rapport aux résultats des autres séries de la littérature (50 à 75\%) [5,9].

Le pronostic maternel de la PG est généralement bénin [13]. A long terme, l'évolution est émaillée de récidives lors des grossesses ultérieures [5] ou lors de la prise d'œstroprogestatifs [2]. La PG expose à 
un risque accru de prématurité et de faible poids de naissance [4]. Des cas de mort fotale in utero, de détresse neurologique et de malformations fotales ont été rapportés [6].

Des lésions bulleuses transitoires sont observées chez 5 et $10 \%$ des nouveaux nés de mères atteintes de PG [2]. La biopsie cutanée et l'IFD ne sont pas nécessaires chez le nouveau-né puisque les lésions évoluent favorablement avec guérison spontanée et définitive sans séquelles avant la fin du premier mois $[2,14]$.

Dans notre série, nous avons rapporté un taux de prématurité de $4,5 \%$ et d'hypotrophie de 6,8\%. De plus, des lésions bulleuses néonatales ont été observées dans notre série chez $4,5 \%$ des cas.

\section{CONCLUSION}

La PGest une DBAI rare nécessitant un diagnostic précoce et une prise en charge multidisciplinaire. Nos résultats confirment ceux rapportés dans la littérature avec la particularité:

- de la fréquence élevée de l'atteinte du visage.

- la rareté de l'atteinte des faces palmo-plantaires.

- Le cas exceptionnel de la PG postabortum.

\section{REFERENCES}

1. Al-Fouzan AW, Galadari I, Oumeish I, Oumeish O. Herpes gestationis (Pemphigoid gestationis). Clinics Dermatol. 2006;24:109-12.
2. Laugel V, Escande B, Donato L, Aberkane K, Heid E, Messer J. Pemphigoïde gravidique et lésions bulleuses chez le nouveau-né. Arch Pédiatr. 2001;8:1071-4.

3. Kroumpouzous G, Cohen M. Specific dermatoses of pronancy: An evidence-based systematic review. Am J Obstet Gynecol. 2003;188:1083-92.

4. Mokni M, Fourati M, Karoui I, El Euch D. Pemphigoïde gravidique étude de 20cas. Ann Dermatol Vénérol. 2004;131:953-6.

5. Boudaya S, Turki H, Meziou TJ, Marrekchi S, Bouassida S, Zahaf A. Pemphigoïde gestationis: une étude de 15 cas. J Gynecol Obstet Biol Reprod. 2003;32:30-4.

6. Triki S, Zeglaoui F, Fazaa B, Mokhtar I, Kamoun MR. La Pemphigoïde Gravidique. A propos de 30 cas. Tunisie Méd. 2006;84:275-8.

7. Engineer L, Bolh K, Ahmed AR. Pemphigoid gestationis: a review. Am J Obstet Gynecol. 2000;83:483-91.

8. Schmutz JL. Dermatoses spécifiques de la grossesse. Press Med. 2003;32:1813-7.

9. Shornik JK. Herpes Gestationis. J Am Acad Dermatol. 1987;17:539-56.

10. Holmes RC, Black MM, Dann J, James DC, Bohogal B. A comparative study of toxic erythema of pregnancy and herpes gestationis. Br J Dermatol. 1982;106:499-510.

11. Kelly SE, Cerio R, Bhogal BS, Black MM. The distribution of IgG subclasses in pemphogoid gestationis PG factors in an IgG1 autoantibody. J Invest Deramtol. 1989;92:695-8.

12. Sitaru C, Powell J, Messer G, Bröcker EB, Wojnarowska F, Zillinkous D. Immunoblotting assay for the diagnosis of pemphigoid gestationis. Obstet Gynecol. 2004;103:757-63.

13. Nguyen T, Alraqum E, Razzaque Ahmed A. Positive clinical outcome with IVIg as monotherapy in recurrent pemphigoid gestationins. Int Immunopharmacol. 2015;26:1-3.

14. Huilaja L, Mäkikallio K, Sormunen R, Lohi J, Hurskainen T, Tasanen K. Gestational pemphigoid: placental morphology and function. Acta Derm Venereol. 2013;93:33-8.

Copyright by Amina Aounallah, et al. This is an open-access article distributed under the terms of the Creative Commons Attribution License, which permits unrestricted use, distribution, and reproduction in any medium, provided the original author and source are credited.

Source of Support: Nil, Conflict of Interest: None declared. 